\title{
Quantitative assessment of the effectiveness of joint measures led by the Fangcang shelter hospitals in the response of COVID-19 epidemic in Wuhan, China
}

Hui Jiang

Beijing Chest Hospital

Pengfei Song

Xi'an Jiaotong University

Siyi Wang

Renmin University of China

Shuangshuang Yin

Xi'an Jiaotong University

Jinfeng Yin

Beijing Chest Hospital

Chendi Zhu

Beijing Chest Hospital

Chao Cai

Beijing YouAn Hospital

Wangli Xu

Renmin University of China

Weimin Li ( $\sim 1711362459 @ q q . c o m$ )

Beijing Chest Hospital

\section{Research Article}

Keywords: COVID-19, shelter hospitals, diagnosis

Posted Date: December 16th, 2020

DOl: https://doi.org/10.21203/rs.3.rs-117120/v1

License: (c) (1) This work is licensed under a Creative Commons Attribution 4.0 International License.

Read Full License 


\section{Title Page}

Full Title: Quantitative assessment of the effectiveness of joint measures led by the Fangcang shelter hospitals in the response of COVID-19 epidemic in Wuhan, China

Running Title: Quantitative assessment the Fangcang shelter hospitals

Hui Jiang*1,2, Pengfei Song*3, Siyi Wang*4, Shuangshuang Yin3, Jinfeng Yin1, Chendi Zhu1,2, Chao Cai5, Wangli Xu†4, Weimin $\mathrm{Li} \uparrow 1,2,6$

1. Beijing Chest Hospital, Capital Medical University, Beijing, 101149, China.

2. Beijing Tuberculosis and Thoracic Tumor Research Institute, Beijing, 101149, China.

3. School of Mathematics and Statistics, Xi'an Jiaotong University, Xi'an, ShaanXi, 710049, China.

4. School of statistics, Renmin University of China, Beijing, 100872, China.

5. Beijing Youan Hospital, Capital Medical University, Beijing, 100069, China.

6. Beijing Municipal Key Laboratory of Clinical Epidemiology, School of Public Health, Capital Medical University, Beijing, 100069, China.

Co-corresponding author: Prof. Weimin Li, Prof. Wangli Xu

Email:1wm_18@aliyun.com,xwlbnu@163.com

Tel \& Fax: 86-10-8950-9359

* Contributed equally to this work.

$\dagger$ Co-senior authors on this study.

Word count (main text): 3,272

The number of tables: 1

The number of figures: 6

The number of appendices: 1 


\section{Abstract}

Objective: To quantitatively evaluate the effectiveness of Fangcang shelter hospitals, designated hospitals, and time interval from illness onset to diagnosis in the prevention and control of COVID19 epidemic.

Methods: We use SEIAR and SEIA-CQFH warehouse models to simulate the two-stage epidemic in Wuhan and calculate the time dependent basic reproduction number (BRN) of symptomatic infected individuals, asymptomatic infected individuals, exposed individuals and community isolated infected individuals. Scenarios that varied in the maximum numbers of open beds in Fangcang shelter hospitals and designated hospitals, the intervals from onset to visit hospitals and diagnosis, are considered to quantitatively assess the optimal measures.

Findings: The BRN is decreased from 4.50 on Jan 22 to 0.18 on March 18. Without Fangcang shelter hospitals, the case number of cumulative and death will increase by $18.58 \%$ and $51.73 \%$. If the number of beds in the designated hospitals decrease by $1 / 2$ or $1 / 4$, the number of cumulative cases will increase by $178.04 \%$ and $92.1 \%$. If the time interval from illness onset to hospital visit is 4 days, the number of cumulative cases and deaths will increase by $2.79 \%$ and $6.19 \%$. If Fangcang shelter hospitals are not established, the number of beds in designated hospitals reduce $1 / 4$ and the time interval from visiting hospitals to diagnosis is 4 days, the cumulative number of cases will increase $268.97 \%$.

Interpretation: The declining BRNs indicate the high effectiveness of joint measures. Joint measures led by Fangcang shelter hospitals play an indispensable contribution and deserves to be rolled out globally, especially when the medical resources are limited. 


\section{Strenghts and limitations of this study}

- Our study provides a detailed quantitative assessment the effect of the Fangcang shelter hospitals, designated hospitals, and time intervals from illness onset to hospital visit and diagnosis of COVID-19 in Wuhan city of mainland China, especially the role of Fangcang shelter hospitals.

- This study did not quantitatively assess the effectiveness of the community isolation and quarantine points isolation due to the difficulty for collection the related data set.

- Unable to collect the real-time number of beds in the central isolation point, and we used the fixed number published by the National Health and Planning Commission. 


\section{Introduction}

In December 2019, coronavirus disease 2019 (COVID-19) outbreak in Wuhan, China. On Jan 31, 2020, World Health Organization (WHO) declared COVID-19 outbreak a public health emergency of international concern ${ }^{1}$. In view of the outbreak of COVID-19 in many countries around the world, WHO announced that the COVID-19 was a global pandemic ${ }^{1}$. In the early stage of the epidemic in Wuhan, thousands of cases rushed to hospitals, and the surge of infections placed huge pressure on the city's medical system ${ }^{2}$. In order to deal with the serious situation of COVID-19, the Chinese government has adopted strategies of joint prevention and control by means of triage, mild/moderate cases and close contacts were isolated at community and quarantine points, and severe and critical cases were admitted to designated hospitals.

Through the implementation of multiple prevention and control measures such as community isolation, quarantine point isolation, designated hospitals, and new diagnostic and intervention techniques, the rational allocation of medical resources and services is guaranteed during COVID19 epidemic. However, there were still huge severity on treatment pressure, like lack of beds and medical resources. In order to relieve pressure, 86 designated hospitals providing about 24,000 beds were rebuilt and new-rebuilt ${ }^{3}$, and a total of 344 national medical teams had been dispatched, with 42,322 medical staffs ${ }^{4}$. In addition, from Feb 5, Wuhan has successively established and opened 16 Fangcang shelter hospitals providing about 13,000 beds to enroll mild/moderate cases. The implementation of multistage joint measures and multi-sectoral division of labor and cooperation have played an important role in the response to the COVID-19. However, although the previous studies have described the definition and function of the Fangcang shelter hospitals ${ }^{2}$, there is no quantitative evaluation on its role in the joint measures. Additionally, there is no study to 
quantitatively evaluate the role of joint measures such as establishing Fangcang shelter hospitals, expanding designated hospitals, shortening the time interval from onset to diagnosis in the response of COVID-19 epidemic.

This study mainly evaluates the effectiveness of joint measures led by the Fangcang shelter hospitals after the closure of Wuhan on Jan $23^{3}$. In addition, in order to include the asymptomatic individuals with infectiousness, we extend the classic SEIR (suspected-exposed-infected- recovered) transmission model to SEIAR model to describe the epidemiological characteristics. Four additional compartments (community isolation (C), quarantine point isolation (Q), Fangcang shelter hospitals (F) and designated hospitals (H)) are added to quantitatively assess the Fangcang shelter hospitals for COVID-19 epidemic in Wuhan from Jan 23 to March 18.

\section{Methods}

\section{Data sources and collection}

From the National Health Commission of the People's Republic of China and WHO we collect the number of newly confirmed cases and cumulative cases, cumulative confirmed cases, and deaths of COVID-19 in Wuhan from Jan 23 to March $18^{1,5}$. The number of the maximum open beds of Fangcang shelter hospitals are collected from Wuhan Municipal Health Commission ${ }^{3}$ (Supplementary Figure 1A). The number of the maximum open beds in designated hospitals were obtained from Wuhan Municipal Health Commission ${ }^{3}$ (Supplementary Figure 1 B). The number of the maximum open beds of quarantine points is obtained from Wuhan Municipal Health Commission ${ }^{3}$.

\section{Data analysis}




\section{SEIAR and SEIAR-CQFH model to stimulate two-stage epidemic in Wuhan}

We use the date of Wuhan locked down to divide the Wuhan epidemic into two time periods, i.e., 2019 Dec 7 to 2020 Jan 22, and Jan 23 to March 18, and use SEIAR and SEIA-CQFH warehouse models to simulate the two-stage Wuhan epidemic, respectively. For the first stage epidemic from Dec 7 to Jan 22, we extend the basic SEIR (suspected-exposed-infected-recovered) model to SEIAR (suspected-exposed-symptomatic infected-asymptomatic infected-recovered) model by enrolling the asymptomatic infected individuals as follows (Figure 1A-B) ${ }^{6}$.

$$
\left\{\begin{array}{l}
\frac{\mathrm{d} S}{\mathrm{dt}}=-\beta_{0}\left(I+f_{A} A+f_{E} E\right) \frac{S}{N}, \\
\frac{\mathrm{d} E}{\mathrm{dt}}=\beta_{0}\left(I+f_{A} A+f_{E} E\right) \frac{S}{N}-\sigma E, \\
\frac{\mathrm{d} I}{\mathrm{dt}}=\rho \sigma E-\gamma_{I} I-\alpha_{I} I, \\
\frac{\mathrm{d} A}{\mathrm{dt}}=(1-\rho) \sigma E-\gamma_{A} A, \\
\frac{\mathrm{d} R}{\mathrm{dt}}=\gamma_{I} I+\gamma_{A} A,
\end{array}\right.
$$

where $S(t), E(t), I(t), A(t), R(t)$ and $N(t)=S(t)+E(t)+I(t)+A(t)+R(t)$ are the number of susceptible, exposed, symptomatic infectious, asymptomatic infectious, recovered individuals and total population in Wuhan at time $t$, respectively. The functions $S(t), E(t), I(t)$ $A(t), R(t), N(t)$ dependent on $t$ are simply denoted as $S, E, I, A, R, N$ in SEIAR model.

For the second stage epidemic from Jan 23 to March 18, we extend SEIAR model to enroll clinically diagnosed cases in community isolation (C), quarantine points isolation (Q), Fangcang shelter hospitals $(\mathrm{F})$ and designated hospitals $(\mathrm{H})$. The SEIAR-CQFH model is described as follows (Figure $1 \mathrm{C})$ : 


$$
\left\{\begin{array}{l}
\frac{\mathrm{d} S}{\mathrm{dt}}=-\beta(t)\left(I+f_{A} A+f_{E} E+f_{C} C\right) \frac{S}{N}, \\
\frac{\mathrm{d} E}{\mathrm{dt}}=\beta(t)\left(I+f_{A} A+f_{E} E+f_{C} C\right) \frac{S}{N}-\sigma E, \\
\frac{\mathrm{d} I}{\mathrm{dt}}=\rho \sigma E-\gamma_{I} I-\alpha_{I} I-\delta I, \\
\frac{\mathrm{d} A}{\mathrm{dt}}=(1-\rho) \sigma E-\gamma_{A} A, \\
\frac{\mathrm{d} C}{\mathrm{dt}}=\delta I-\gamma_{C} C-C_{H}(t)-C_{Q}(t)-C_{F}(t), \\
\frac{\mathrm{d} Q}{\mathrm{dt}}=C_{Q}(t)-\left(1-\rho_{Q}\right) \gamma_{Q} Q-Q_{F}(t)-Q_{H}(t), \\
\frac{\mathrm{d} F}{\mathrm{dt}}=Q_{F}(t)+C_{F}(t)-\left(1-\rho_{F}\right) \gamma_{F} F-F_{H}(t), \\
\frac{\mathrm{d} H}{\mathrm{dt}}=C_{H}(t)+Q_{H}(t)+F_{H}(t)-\gamma_{H} H-\alpha_{H} H, \\
\frac{\mathrm{d} R}{\mathrm{dt}}=\gamma_{I} I+\gamma_{A} A+\gamma_{C} C+\left(1-\rho_{Q}\right) \gamma_{Q} Q+\left(1-\rho_{F}\right) \gamma_{F} F+\gamma_{H} H,
\end{array}\right.
$$

where $S(t), E(t), I(t), A(t), R(t), N(t)$ have the same meanings as that in SEIAR model, and $C(t), Q(t), F(t), H(t)$ are the number of community isolation, quarantine points isolation, Fangcang shelter hospitals and designated hospitals at time $t$, respectively. More details about the model parameters and functions setting can be found in supplementary.

A

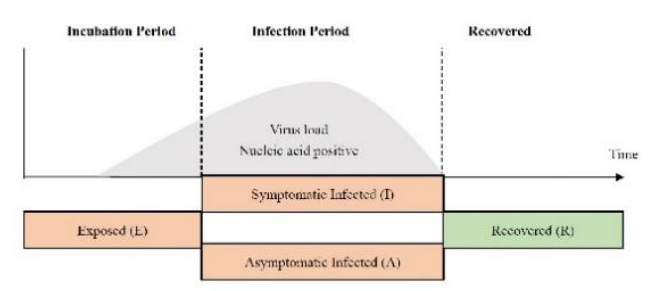

C

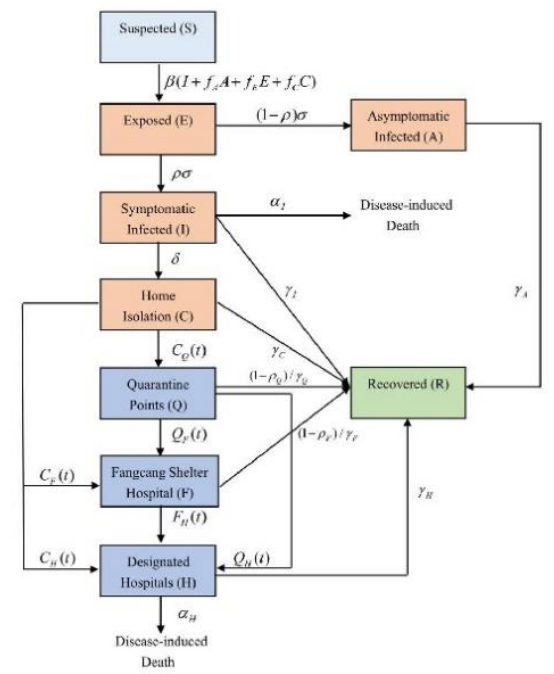

Figure 1: Descriptions of the first and second stages by SEIAR and SEIAR-CQFH models 
Panel A: Epidemiological descriptions of exposed (E), symptomatic infectious (I) and asymptomatic infectious (A) individuals

Panel B: Descriptions of the first stage (Dec 7 to Jan 22) by SEIAR model

Panel C: Descriptions of the second stage (Jan 23 to March 18) by SEIAR-CQFH model

Some of the parameters are determined from existing references (See Table 1 for detailed description) and seven parameters $\left(\beta_{0}, \beta_{\text {end }}, r, \alpha_{I}, \alpha_{A}, \delta_{c q}, \theta\right)$ are unknown and estimated by the nonlinear least-square(NLES) method on the basis of newly confirmed, cumulative confirmed and cumulative COVID-19 death cases in Wuhan from Jan 23 to March 18. The confidence interval of the parameters is calculated by stochastic simulation method (Table 1).

Table 1. Descriptions of parameters used in SEIAR and SEIAR-CQFH models

\begin{tabular}{|c|c|c|c|}
\hline Parameter & Implication & Value & Sources \\
\hline$\beta_{0}$ & The transmission rate of symptomatic infectious before Jan 23 & $\begin{array}{l}0.43 \text { (95\%CI: } 0.428- \\
0.431)\end{array}$ & Estimated \\
\hline$\beta_{\text {end }}$ & $\begin{array}{l}\text { The minimum transmission rate of symptomatic infectious under } \\
\text { interventions after Jan } 23\end{array}$ & $\begin{array}{l}0.05(95 \% \mathrm{CI}: 0.049- \\
0.051)\end{array}$ & Estimated \\
\hline$r$ & $\begin{array}{l}\text { Exponential decreasing rate of transmission rate of symptomatic infectious } \\
\text { under interventions after Jan } 23\end{array}$ & $\begin{array}{l}0.072(95 \% \mathrm{CI}: \\
0.0715-0.0728)\end{array}$ & Estimated \\
\hline$f_{E}$ & The fitness of transmission rate with respect to exposed individuals & $1 / 3$ & Ref 7 \\
\hline$f_{A}$ & The fitness of transmission rate with respect to asymptomatic infectious & $1 / 3$ & Ref 7 \\
\hline$f_{C}$ & $\begin{array}{l}\text { The fitness of transmission rate with respect to home isolated symptomatic } \\
\text { infectious }\end{array}$ & 1 & Assumed \\
\hline $1 / \sigma$ & Incubation period & 5.2 & Ref $8-10$ \\
\hline$\rho$ & $\begin{array}{l}\text { The probability that each exposed individual } \\
\text { enters the symptomatic compartments }\end{array}$ & 0.82 & Ref 11 \\
\hline $1 / \gamma_{I}$ & The average duration of the infection of symptomatic infectious & 12 & Ref 10 \\
\hline $1 / \gamma_{A}$ & The average duration of the infection of asymptomatic infectious & 7 & Ref 10 \\
\hline
\end{tabular}




\begin{tabular}{|c|c|c|c|c|}
\hline $1 / \gamma_{C}$ & $\begin{array}{l}\text { The average duration of the infection of Home isolated symptomatic } \\
\text { infectious }\end{array}$ & 11 & & Ref 10 \\
\hline $1 / \gamma_{Q}$ & The average isolated period of Quarantine isolated symptomatic infectious & 14 & & Ref 10 \\
\hline $1 / \gamma_{F}$ & $\begin{array}{l}\text { The average hospital day of symptomatic infectious in Fangcang shelter } \\
\text { hospitals }\end{array}$ & 20 & & Ref 10 \\
\hline $1 / \gamma_{H}$ & The average hospital day of symptomatic infectious in Hospitals & 10 & & Ref 10 \\
\hline$\alpha_{I}$ & Disease-induced death rate of symptomatic infectious estimated & $\begin{array}{l}0.0087(95 \% \mathrm{CI}: \\
0.007-0.01)\end{array}$ & & Estimated \\
\hline$\alpha_{H}$ & Disease-induced death rate of symptomatic infectious in hospitals & $\begin{array}{l}0.0057(95 \% \mathrm{CI}: \\
0.0051-0.0061)\end{array}$ & & Estimated \\
\hline $1 / \delta$ & The average day from symptom onset to clinical diagnosed after Jan 23 & 1 & & Ref 10 \\
\hline $1 / \delta_{c f h}$ & The average day from clinical diagnosed to laboratory confirmed & 1 & & Ref 10 \\
\hline$\delta_{c q}$ & The moving rate from Community isolation to Quarantine points isolation & $\begin{array}{l}0.2(95 \% \mathrm{CI}: \\
0.21)\end{array}$ & $0.19-$ & Estimated \\
\hline$\rho_{Q}$ & $\begin{array}{l}\text { The deteriorating rate from mild or moderate to severe illness in Quarantine } \\
\text { points }\end{array}$ & 0.2 & & Ref 12 \\
\hline$\rho_{F}$ & $\begin{array}{l}\text { The deteriorating rate from mild or moderate to severe illness in Fangcang } \\
\text { shelter hospitals }\end{array}$ & 0.05 & & Ref 12 \\
\hline$\theta$ & $\begin{array}{l}\text { The ratio of the beds used to isolate symptomatic infectious individuals in } \\
\text { Quarantine points }\end{array}$ & $\begin{array}{l}0.09(95 \% \mathrm{CI}: \\
0.11)\end{array}$ & $0.08-$ & Estimated \\
\hline
\end{tabular}

Note: Reference $=$ Ref

\section{Basic reproduction number for exposed, asymptomatic, symptomatic, and community}

\section{isolated infected individuals}

The basic reproduction number, defined as the expected average number of secondary cases produced in a completely susceptible population by a typical infective individual during the infectious period, is one of the most significant concepts in population biology ${ }^{13,14}$. More importantly, it often determines the threshold behavior for many epidemic models. It is often the case that a disease will be died out if the basic reproduction number is less than unity and the disease will be established in the population if it is greater than unity. Hence, it is a commonly used to measure of the effort for controlling an infectious disease in epidemiology. We apply the next 
generation matrix to estimate the basic reproduction number $R_{0}(t)$ with control measures in forcing as follows:

$\mathrm{R}_{0}(t)= \begin{cases}\frac{\rho \beta_{0}}{\gamma_{I}+\alpha_{I}}+\frac{f_{E} \beta_{0}}{\sigma}+\frac{(1-\rho) f_{A} \beta_{0}}{\gamma_{A}}, & \text { Before Jan 23, } \\ \frac{\rho \beta(t)}{\gamma_{I}+\delta+\alpha_{I}}+\frac{f_{E} \beta(t)}{\sigma}+\frac{(1-\rho) f_{A} \beta(t)}{\gamma_{A}}+\frac{f_{C} \rho \delta \beta(t)}{\left(\left(\gamma_{I}+\alpha_{I}+\delta\right)\left(\gamma_{C}+\left(C_{Q}(t)+C_{F}(t)+C_{H}(t)\right) / C(t)\right)\right.}, & \text { After Jan 23. }\end{cases}$

In addition, the basic reproduction number of exposed, asymptomatic, symptomatic, and community isolated infected cases are respectively as:

$\mathrm{R}_{0}^{E}(t)=\left\{\begin{array}{ll}\frac{f_{E} \beta_{0}}{\sigma}, & \text { Before Jan 23, } \\ \frac{f_{E} \beta(t)}{\sigma}, & \text { After Jan 23, }\end{array} \mathrm{R}_{0}^{A}(t)=\left\{\begin{array}{ll}\frac{(1-\rho) f_{A} \beta_{0}}{\gamma_{A}}, & \text { Before Jan 23, } \\ \frac{(1-\rho) f_{A} \beta(t)}{\gamma_{A}}, & \text { After Jan 23, }\end{array} \mathrm{R}_{0}^{I}(t)= \begin{cases}\frac{\rho \beta_{0}}{\gamma_{I}+\alpha_{I}}, & \text { Before Jan 23, } \\ \frac{\rho \beta(t)}{\gamma_{I}+\delta+\alpha_{I}}, & \text { After Jan 23, }\end{cases}\right.\right.$ and

$$
\mathrm{R}_{0}^{C}(t)=\frac{f_{C} \rho \delta \beta(t)}{\left(\left(\gamma_{I}+\alpha_{I}+\delta\right)\left(\gamma_{C}+\left(C_{Q}(t)+C_{F}(t)+C_{H}(t)\right) / C(t)\right)\right.}, \text { After Jan } 23
$$

The estimation of the basic reproduction numbers and their confidence intervals is calculated from the above formulas based on the estimated 1,000 groups of values.

\section{Patient and Public Involvement}

Patients were not involved in the design of this study.

\section{Results}

\section{SEIAR and SEIA-CQFH models simulated two-stage epidemics}

The number of newly confirmed cases, cumulative confirmed and death cases $(0,50,005$ and 2,496) until March 18 reported in Wuhan are basically consistent with the models simulated results (21, 50,926 and 2,590), which show that real data and predicted values showed the model simulate well

(Figure 2A-C). In addition, after taking various prevention and control measures, although the BRN fluctuated slightly from February 3 to February 9, the overall trend is that the BRN was decreased 
from 4.50 on Jan 22 to 0.18 on March 18. Specifically, the BRN of symptomatic infected individuals, asymptomatic infected individuals and exposed individuals decreased from 3.57, 0.18 and 0.75 to 0.04, 0.02 and 0.10 from Jan 23 to March 18. In addition, although the BRN of community isolation symptomatic infected individuals increased slightly from 0.24 on Jan 24 to 0.41 on Feb 2, the BRN has continued to decrease from 0.27 on Feb 8 to 0.02 on March 18 (Figure 2D). In addition, SEIARCQFH model simulates the transmission rule of epidemic after Jan 23, and find that the increase range of number of cumulative confirmed cases and death cases is smooth, except for Feb 12 (Figure 2A). The reason of surge is that on Feb 12 clinical diagnosis cases were enrolled as the confirmed cases. 
A
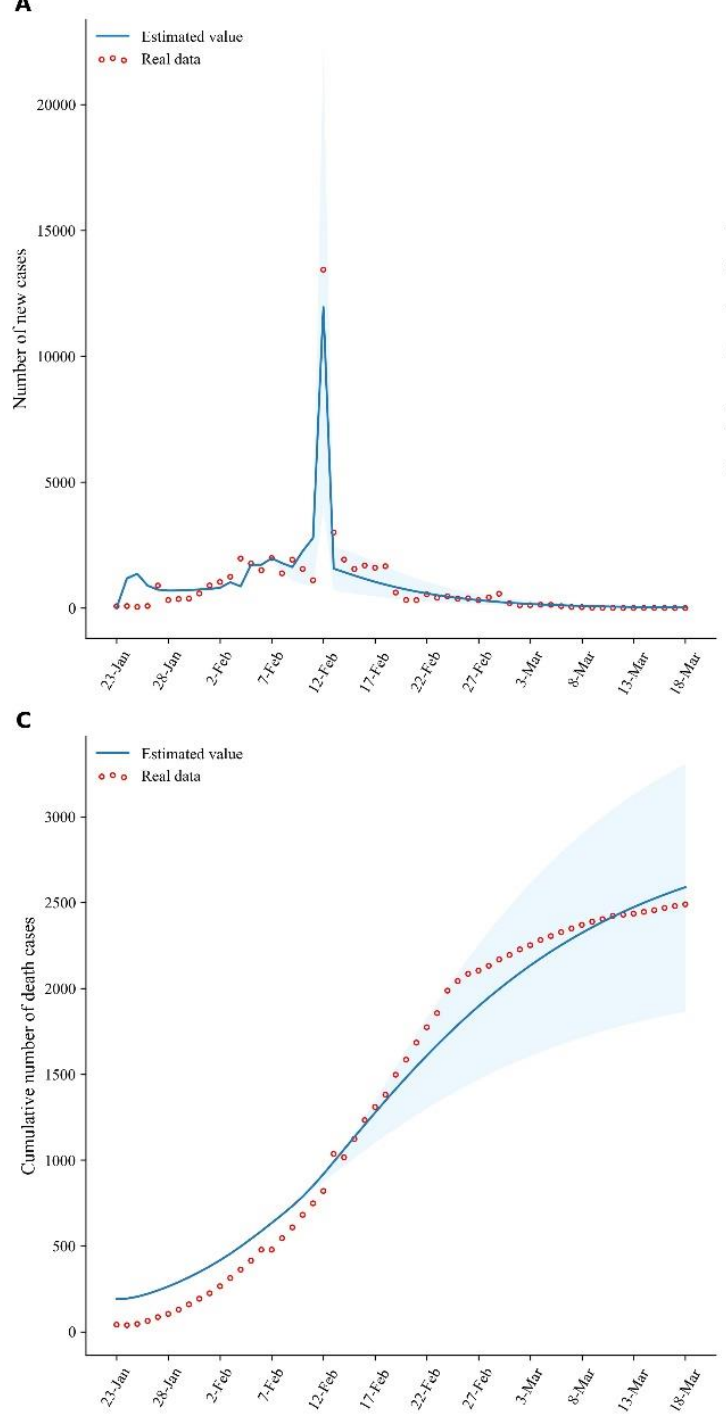

B

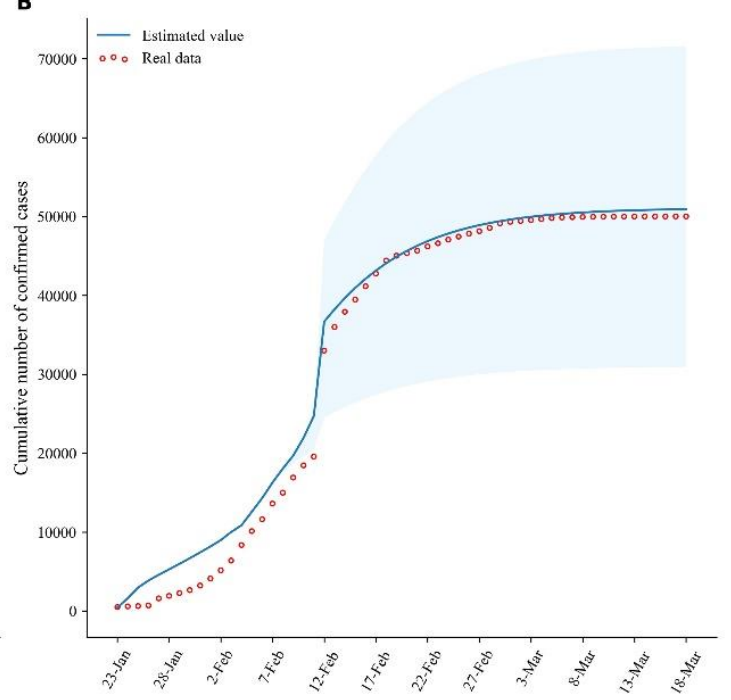

D

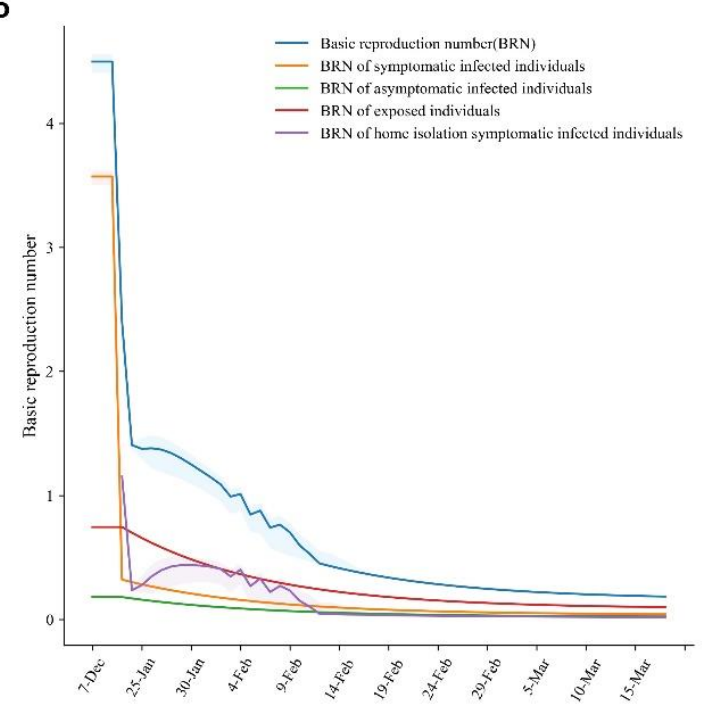

Figure 2: The number of COVID-19 cases and the basic reproductive number (BRN) simulated by SEIAR and SEIAR-CQFH models

Panel A: Comparison of the number of new cases simulated by model and real data

Panel B: Comparison of the cumulative number of cases simulated by model and real data

Panel C: Comparison of the number of deaths simulated by model and real data

Panel D: The BRN of different types of cases

Assessment of Fangcang shelter hospitals

SEIAR-CQFH model simulates the beds in Fangcang shelter hospitals, and we can see if the number 
of beds was reduced by $1 / 2$ or $3 / 4$, the growth ranges of number of cumulative confirmed cases and death cases increase obviously, especially the cumulative deaths. Specifically, if the number of beds in Fangcang shelter hospitals is $0,1 / 4,1 / 2$ and normal, the cumulative number of cases on March 18 are 60,389, 56,924, 54,430 and 50,925 (real data: 50,005), respectively. By March 18, there will be $18.58 \%, 11.78 \%$ and $6.88 \%$ more cumulative confirmed cases in $0,1 / 4$ and $1 / 2$ beds, compared to the condition with normal beds. (Figure 3A). Compare to the cumulative number (425) of cases on Jan 23, month-on-month growth in $0,1 / 4,1 / 2$ and normal beds are $12533.73 \%, 12082.60 \%$, $11683.11 \%$ and $11049.31 \%$, respectively (Figure $3 \mathrm{~A}$ ). In addition, if the number of beds in Fangcang shelter hospitals is $0,1 / 4,1 / 2$ and normal, the number of deaths on 18 March are 3,929, 3,399, 3,019 and 2,590 (real data: 2,495), respectively. By 18 March, there will be 51.73\%, 31.25\% and $16.59 \%$ more cumulative death cases in $0,1 / 4$ and $1 / 2$ beds, compare to the condition with normal beds. Compare to the death number (190) on Jan 23, month-on-month growth in 0, 1/4, 1/2 and normal beds are $954.56 \%, 927.92 \%, 872.48 \%$ and $779.35 \%$, respectively (Figure 3B).
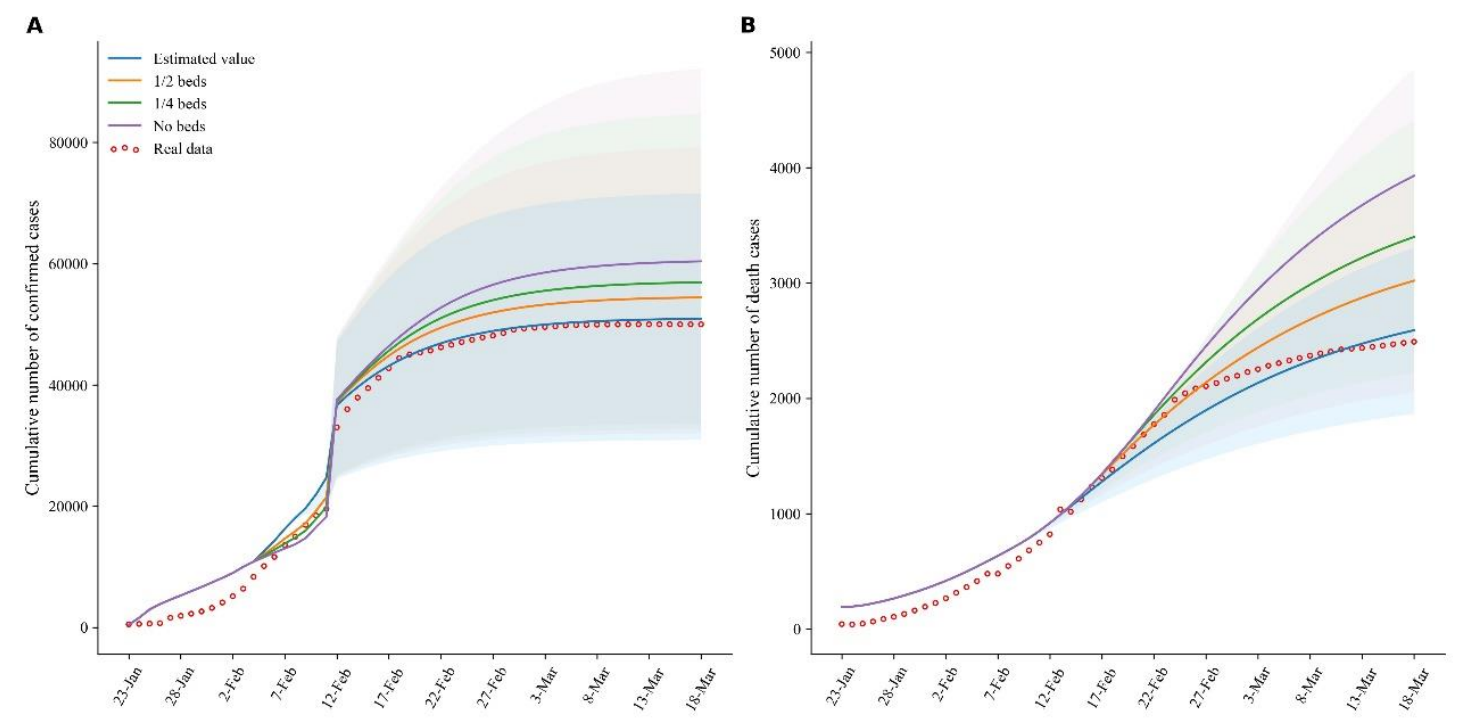

Figure 3: The cumulative number of COVID-19 cases and deaths varies with the number of beds in Fangcang shelter hospitals 


\section{Panel A: The cumulative number of COVID-19 cases}

\section{Panel B: The cumulative number of COVID-19 deaths}

\section{Assessment of designated hospitals}

The number of beds in designated hospitals has a similar impact as that in Fangcang shelter hospitals on the COVID-19 epidemic, however, it is important to note that reducing the number of beds in designated hospitals will lead to a more significant increase in confirmed cases. Specifically, if the number of beds in designated hospitals is $1 / 4,1 / 2$ and normal, the cumulative number of confirmed cases of 18 March are 141,594, 97,829 and 50,926 (real data: 50,005), respectively. By 18 March, there will be $178.04 \%$ and $92.1 \%$ more cumulative confirmed cases in $1 / 4$ and $1 / 2$ beds, compare to the condition with normal beds. Compare to the cumulative number of cases (425) on Jan 23, monthon-month growth in $1 / 4,1 / 2$ and normal beds are $23234.81 \%, 18784.59 \%$ and $11049.31 \%$. Although the number of cases increased slightly with the increase of beds size in the early period, the increase in the number of beds significantly inhibited the increase in the number of cases in the later period (Figure 4). 


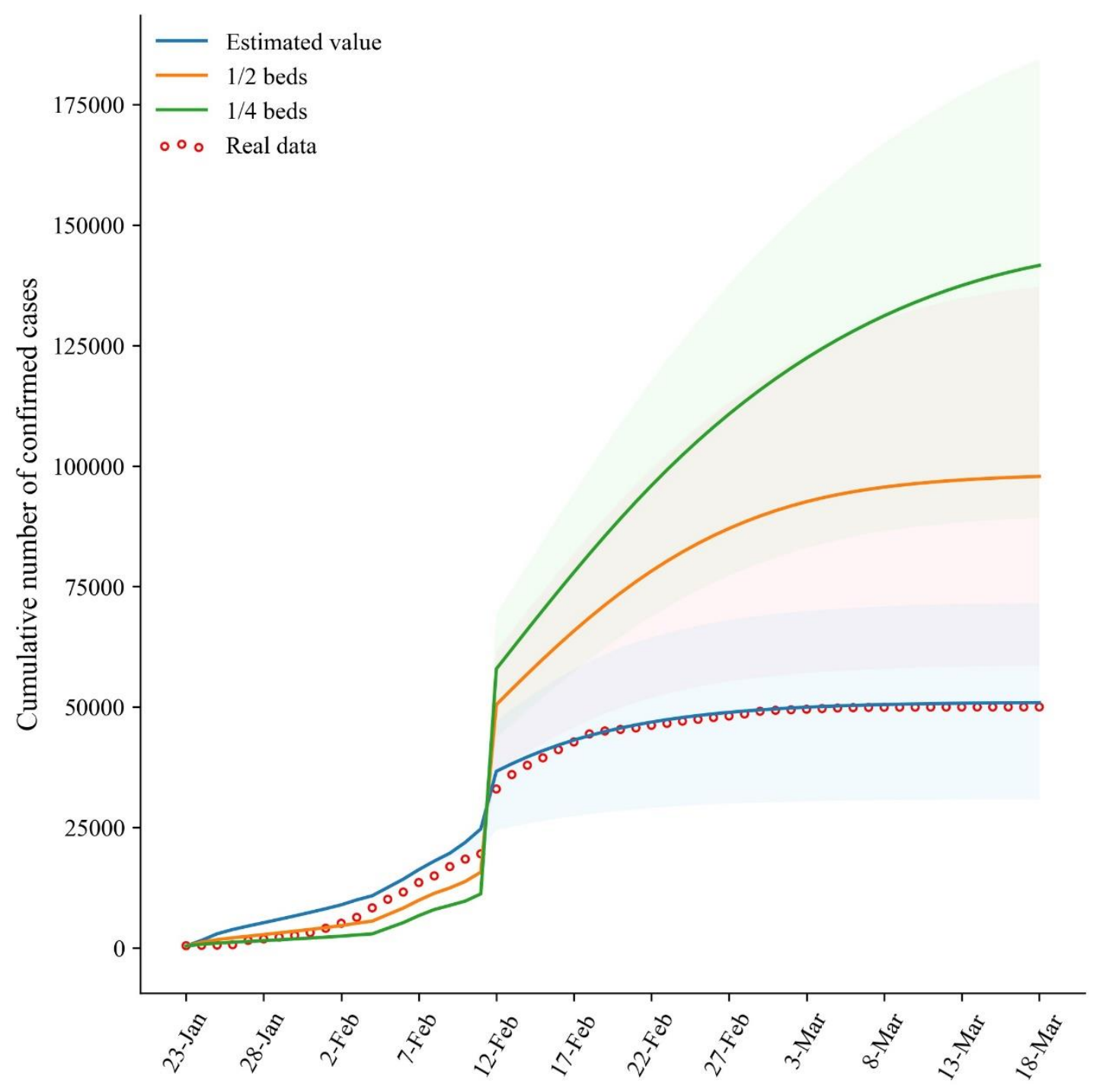

Figure 4: The cumulative number of COVID-19 cases varies with the number of beds in designated hospitals

\section{Assessment of the joint measures led by the Fangcang shelter hospitals}

we use SEIAR-CQFH model to estimate the impact of time interval from onset to visit hospital of 1 day, 2 days and 4 days on the COVID-19 epidemic and find that the time interval from illness onset to hospital visit is of importance for the epidemic. Compare to 1 and 2 days, the time interval from illness onset to hospital visit is 4 days, resulting in a significant increase in the number of confirmed cases and deaths. Specifically, the cumulative number of cases and deaths as of March 
18 are 54,350 and 2,750 , respectively, an increase of $6.73 \%, 4.29 \%$ and $6.19 \%, 3.31 \%$ compare with that of 1 day and 2 days (Figure 5A-B).

The increased impact of the time interval from hospital visit to diagnosis is more pronounced on the number of confirmed cases and deaths. Specifically, before Feb 12, the time interval from hospital visit to diagnosis has less effect on the cumulative number of cases and deaths. After Feb 12, the shorter the time interval from visit hospital to diagnosis, the fewer cases are accumulated. As of March 18, the cumulative number of cases are 50,926, 55,334 and 64,863, respectively, for 1 day, 2days and 4 days. In addition, the cases are more significantly affected after Feb 22, i.e., the shorter the time interval from hospital visit to diagnosis, the fewer the number of deaths, and the gap increases with the time. As of March 18, the total number of deaths is 2,590 (real data: 2,496), 26,280 and 27,740, respectively (Figure 5C-D). 
A
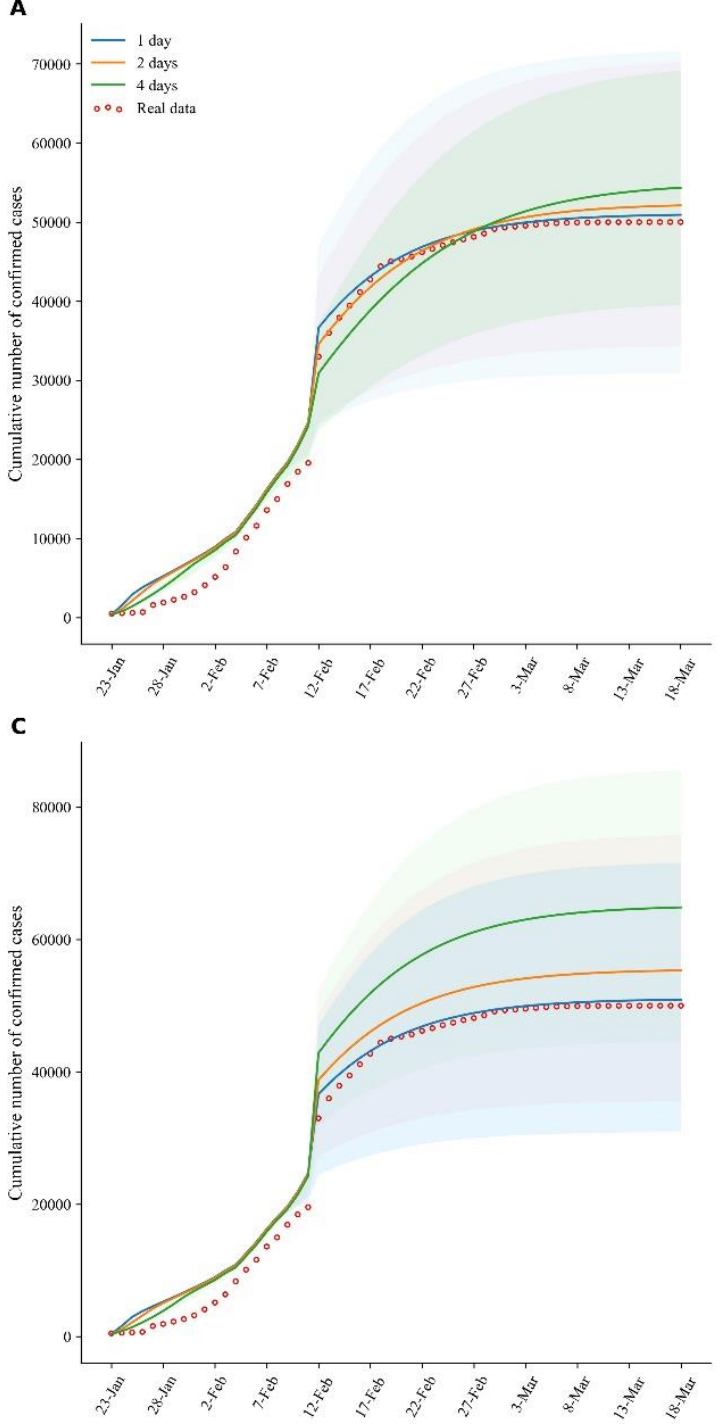

B

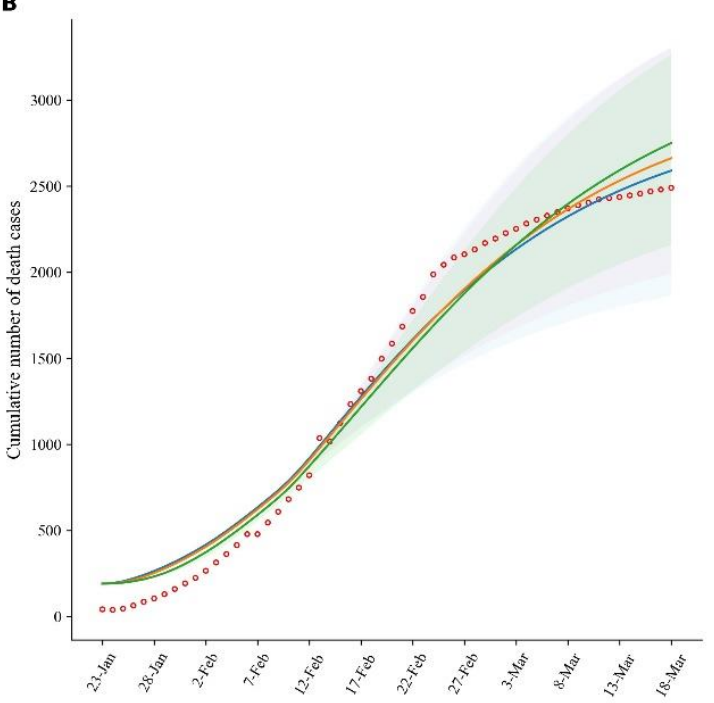

D

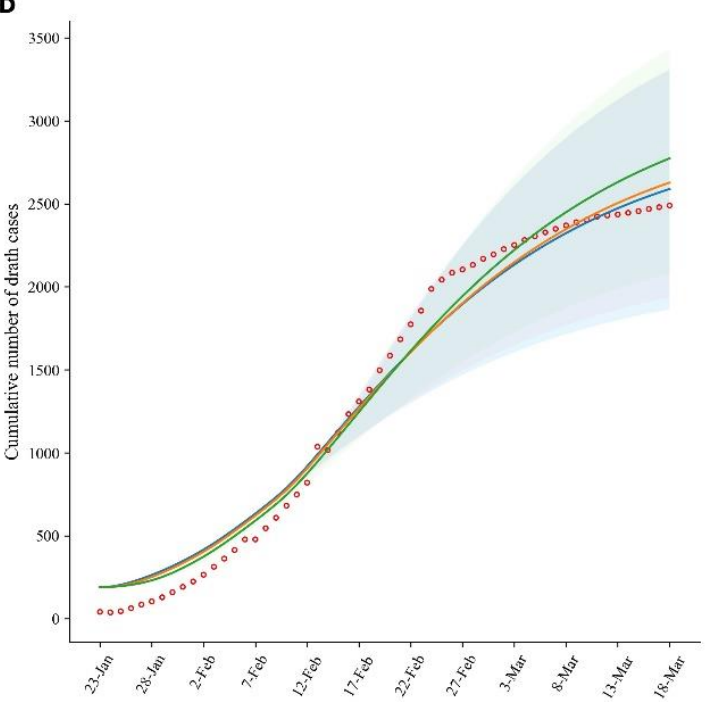

Figure 5: The cumulative number of COVID-19 cases and deaths varies with the time intervals

Panel A: The cumulative number of COVID-19 cases varies with the time intervals from illness onset to hospital visit

Panel B: The cumulative number of COVID-19 deaths varies with the time intervals from illness onset to hospital visit

Panel C: The cumulative number of COVID-19 cases varies with the time intervals from hospital visit to diagnosis

Panel D: The cumulative number of COVID-19 deaths varies with the time intervals from hospital visit to diagnosis 
Generally speaking, in view of the severity and public concern of the COVID-19 epidemic, the onset individuals usually visit hospital soon, other medical services including the time for diagnosis, the number of beds in Fangcang shelter hospitals, and the number of beds in designated hospitals are evaluated and required by the Chinese government. Therefore, we put the dynamic change of the above variables except the variables for onset to visit hospitals into the model to simulate the effect of changes of multiple variables on cumulative number of cases to evaluate the joint measures. The results show that the number of beds in Fangcang hospitals and designated hospitals are normal, and both the time intervals from onset to hospital visit and diagnosis are one day, the cumulative number of cases is the fewest and basically consistent with the real data $(50,926$; real data: 50,005). The number of Fangcang shelter hospitals is zero, the number of beds in designated hospitals is $1 / 4$, the time interval from onset to hospital visit is one day and the time interval from hospital visit to diagnosis is four days, the cumulative number of cases is the most $(187,904)$. For the other combinations' types of medical services, the cumulative number of cases caused are higher than the actual number of prevention and control measures (Figure 6).

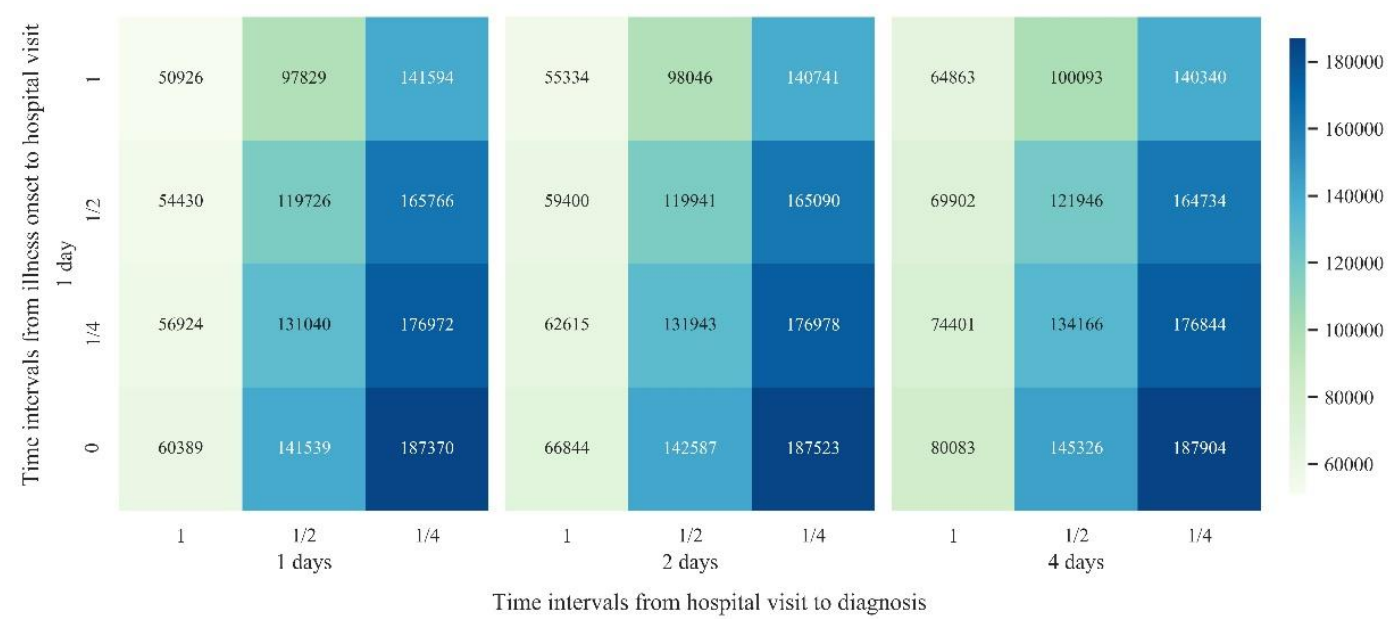

Figure 6: The cumulative number of COVID-19 cases varies with the joint prevention and control measures 


\section{Discussion}

With asymptomatic cases considered in the classic transmission dynamic model SEIR, we simulate the transmission rule of epidemic by an extended SEIAR model before and after Jan 23, we add four cabins, such as community isolation, quarantine point isolation, Fangcang sheleter hospitals, designated hospitals and construct the SEIAR-CQFH model for simulating disease transmission. The simulated results show that the number of new cases $(21)$, cumulative confirmed cases $(50,926)$ and deaths $(2,590)$ until March 18 are estimated by two-stage SEIAR and SEIAR-CQFH models, which are close to the data published by the government $(0,50,005 \text { and } 2,496)^{1,3,5}$. In addition, for any day from Dec 7 to March 18, the real data is matched with the simulated data, which indicates that the two-stage models are appropriate for COVID-19 transmission. Also, the BRN was decreased from 4.50 on Jan 23 to 0.18 on March 18, which is also consistent with other researches ${ }^{15-}$

17. In addition, the BRN of symptomatic infected cases, asymptomatic infected cases, exposed individuals, and isolated infected cases decreased from Jan 23 to March 18. The time when the BRN began to decline was basically the same as the time when the national medical teams assisted Wuhan and the closure of Wuhan ${ }^{18,19}$ and indicates that the effectiveness of joint prevention and control strategies adopted by the Chinese government. Therefore, we use SEIAR-CQFH model to quantitatively assess those measures of Fangcang shelter hospitals, designated hospitals, and time intervals from onset illness to diagnosis in COVID-19 epidemic after Jan 23 in Wuhan city in mainland China.

In the joint prevention and control of the COVID-19 epidemic, Fangcang shelter hospitals play important isolation and triage roles as the intermediate platform. At the beginning of Feb 2020, designated hospitals in Wuhan had not enough beds for COVID-19 patients, especially for 
thousands of patients with mild to moderate COVID-19,3. Mild and moderate patients could be isolated at community, however, epidemiological study showed that in China COVID -19 has a high rate of intrafamily transmission ${ }^{2,10,20-23}$, and more than 50\% COVID-19 patients had at least one family member with the disease ${ }^{2}$. In addition, community isolation is difficult to monitor disease progress, and the asymptomatic infectious individuals may deteriorate to mild and moderate illness $^{21-23}$. Our study also exhibits that the BRN for community isolation symptomatic infected individuals increased from Jan 24 to Feb 2, while other types of BRN was decreasing. This indicates that the effect of community isolation is not good. On Feb 2, Wuhan asked community isolation individuals, newly suspected individuals, and close contacts to designated points isolations. After three days, on Feb 5, 2020, Wuhan has successively opened 16 Fangcang shelter hospitals to treat mild and moderate patients. This implementation of this measure is conducive to the rapid isolation and triage of mild and moderate cases. Therefore, from Feb 5, the BRN of community isolation symptomatic infected individuals shows a continuous downward trend.

Beside isolation, triage, the basic medical care and frequent monitoring and rapid referral were also the original intention of the establishment of the Fangcang shelter hospitals ${ }^{2,3}$. Our study also confirms the important role of the Fangcang shelter hospitals in early treatment the COVID-19 cases, the results show that without Fancang shelter hospitals, the cumulative number of cases and deaths would increase by $18.58 \%$ and $51.73 \%$ by March 18 . In addition, if the number of beds is reduced to $1 / 2$ or $1 / 4$, the cumulative number of cases and deaths would increase by $6.88 \%, 11.78 \%$ and $16.59 \%, 31.25 \%$. Moreover, one of the important functions of the Fancang shelter hospitals was monitoring and rapid referral ${ }^{2}$, which is to enable severe COVID-19 cases to be treated in the shortest time and increase the survival possibility. The treatment of severe cases is inseparable from 
the designated hospitals, designated hospitals also play an important role as the high-level platform in hierarchical prevention and control. Our study also shows that, if the number of beds in the designated hospitals decrease by $1 / 2$ or $1 / 4$, the number of COVID-19 cases will increase significantly (from 50,926 to 97,829 and 141,594). After Jan 25, with progressively increasing medical materials and medical staff, the number of beds in designated hospitals was all expanded, which increased the treatment opportunities of severe cases, reduce the death of severe cases of COVID-19.

Shortening the time interval between hospital transfers can increase the survival possibility of severe cases, similarly, shortening the time intervals from illness onset to hospital visit and confirm can also reduce deaths and transmission. Our study shows that the number of deaths and culminative cases decrease significantly after reducing the time interval from illness onset to hospitals visit and from visit hospital to confirm. We use models to simulate effect of joint measures and find that if the number of beds in Fangcang hospitals and designated hospitals are normal, and both the time intervals from onset to hospital visit and diagnosis are one day, the cumulative number of cases is the fewest and basically consistent with the real data $(50,926$; real data: 50,005$)$. So the measure at that stage was the optimal, If Fangcang shelter hospitals are not established, the number of beds reduce $1 / 4$ and the time interval is 4 days, the cumulative number of cases will increase $268.97 \%$. This further verifies the importance of the joint measures in COVID-19 epidemic prevention and control and deserves to be rolled out globally. In addition, the number of beds in Fangcang shelter hospitals and designated hospitals and the time interval of diagnosis, are the best combination after detailed and professional evaluation by the Chinese government, which is the best and fastest choice after fully evaluating the materials, personnel, and other conditions under the increasingly severe 
situation of the COVID-19 epidemic. Ours study also verifies this result. However, we must think about and sum up how to learn from the COVID-10 epidemic response and improve the ability to deal with emerging infectious diseases, especially when the medical resources are limited.

This study has some limitations. Firstly, this study did not quantitatively assess the effectiveness of the community isolation and quarantine points isolation due to the difficulty for collection the related data set. Secondly, unable to collect the real-time number of beds in the central isolation point, and we used the fix ed number published by the National Health and Planning Commission.

In conclusion, our study provides a detailed quantitative assessment the effect of the Fangcang shelter hospitals, designated hospitals, and time intervals from illness onset to hospital visit and diagnosis of COVID-19 in Wuhan city of mainland China, especially the role of Fangcang shelter hospitals. The results showed that Fangcang shelter hospitals, like designated hospitals, played an irreplaceable contribution to the control of COVID-19 epidemic; moreover, the combination of measures, including the normal number of beds in Fangcang hospitals, is optimum, made the prevention and control strategies more effectiveness. Lastly, although the COVID-19 epidemic has been basically brought under control in China and Fangcang shelter hospitals have been closed, we still cannot take it lightly. We should sum up the prevention and control experience of COVID-19, and provide more scientific methods for Chinese and even global people in response to the outbreak of emerging infectious disease, especially for countries and regions with limited medical resources. 


\section{Declarations}

\section{Ethical approval and consent to participate}

Not applicable.

\section{Consent for publication}

Not applicable.

\section{Availability of data and material}

Our data are from publicly published data, have no privacy implications and can be founded in http://wjw.wuhan.gov.cn/.

\section{Funding}

This study was funded by grants from the National Key Research and Development Program (2018YFC2000300); National Science and Technology Major Project of China (2018ZX10302 302001004); National Natural Science Foundation of China (U1903118); National Natural Science Foundation of China (11631012); National Natural Science Foundation of China (11971478).

\section{Author contributions}

Li W and Xu W conceived, designed, and supervised the study. Song P, Wang S, Zhu C and Cai C collected the data and cleaned the data. Jiang H and Song P analyzed the data. Jiang H and Song P wrote the drafts of the manuscript. Jiang $\mathrm{H}$ and Li W interpreted the findings. Li $\mathrm{W}$ and $\mathrm{Xu} \mathrm{W}$ commented on and revised the drafts of the manuscript. All authors read and approved the final manuscript.

\section{Conflicts of interests}


All authors declare no competing interests.

Acknowledgment

Not applicable. 


\section{Reference}

1. World Health Organization (WHO). Coronavirus disease (COVID-19) Pandemic. https://www.who.int/emergencies/diseases/novel-coronavirus-2019. (Date accessed: May 14, 2020).

2. Chen S, Zhang Z, Yang J, et al. Fangcang shelter hospitals: a novel concept for respon ding to public health emergencies. Lancet 2020; 359: 1305-1314

3. Wuhan Municipal Health Commission. COVID-19.

http://wjw.wuhan.gov.cn/. (Date accessed: May 14, 2020).

4. Sina. Physicians supporting Hubei.

http://blog.sina.com.cn/s/blog_45bb8ce70102yory.html. (Date accessed: May 14, 2020).

5. National Health Commission of the People's Republic of China. COVID-19.

http://www.nhc.gov.cn/. (Date accessed: May 14, 2020).

6. Brauer F, Castillo-Chavez C. Mathematical models in population biology andepidemiolog y. New York: Springer, 2012.

7. Chen Y, Wang A, Yi B, et al. Epidemiological characteristics of infection inCOVID-19 close contacts in Ningbo city (in Chinses). Chin J Epidemiol 2020; 41(5): 667-671.

8. Backer J, Klinkenberg D, Wallinga J. Incubation period of 2019 novel coronavirus (201 9-nCoV) infections among travellers from Wuhan, China, 20-28 January 2020. Eurosurv eillance 2020; 25(5): 200006.

9. Lauer SA, Grantz KH, Bi Q, et al. The incubation period of coronavirus disease 2019 (COVID-19) from publicly reported confirmed cases: estimation and application. Ann Int ern Med 2020; 172(9): 577-582. 
10. Special Expert Group for Control of the Epidemic of Novel Coronavirus Pneumonia o $\mathrm{f}$ the Chinese Preventive Medicine Association. An update on theepidemiological charact eristics of novel coronavirus pneumonia (COVID-19) (in Chinese). Chin J Epidemiol 20 20; 41(2): 139-144.

11. Qiu J. Covert coronavirus infections could be seeding new outbreaks. Nature 2020.

12. Sina. The deteriorating rate from mild or moderate. http://k.sina.com.cn/article_7211561239_mladd7b11703300wsxs.html?from=health. (Date a ccessed: May 14, 2020).

13. Anderson R, May R. Infectious diseases of humans: dynamics and control. Oxford: $O x$ ford University Press, 1992.

14. Diekmann O, Heesterbeek J. Mathematical epidemiology of infectious diseases: model building, analysis and interpretation. Chichester: John Wiley \& Sons, 2000.

15. Sanche S, Lin Y T, Xu C, et al. High Contagiousness and Rapid Spread of Severe A cute Respiratory Syndrome Coronavirus 2. Emer Infect Dis 2020, 26(7).

16. Wu J, Leung $\mathrm{K}$, Leung $\mathrm{G}$. Nowcasting and forecasting the potential domestic and inte rnational spread of the 2019-nCoV outbreak originating in Wuhan, China: a modelling study. Lancet 2020; 395(10225): 689-697.

17. Zhou T, Liu Q, Yang Z, et al. Preliminary prediction of the basic reproduction numbe $\mathrm{r}$ of the Wuhan novel coronavirus 2019-nCoV. J Evid Based Med 2020; 13(1): 3-7.

18. The Central People's Government of the People's Republic of China. Announcement of novel coronavirus infection prevention command headquarters in Wuhan. Website of $H$ ubei Provincial Government 2020. 
http://www.gov.cn/xinwen/2020-01/23/content 5471751.htm. (Date accessed: May 14, 202 $0)$.

19. National Health Commission of the People's Republic of China. National Health and Health Commission organized several medical teams to reinforce Wuhan (News). Website of Hubei Provincial Government 2020. http://www.hubei.gov.cn/zhuanti/2020/gzxxgzbd/gdzs/202001/t20200125 2014882.shtml. (Date accessed: May 14, 2020)

20. Tian S, Hu N, Lou J, et al. Characteristics of COVID-19 infection in Beijing. J Infec 2020; 80(4): 401-406.

21. WHO. Report of the WHO-China Joint Mission on coronavirus disease 2019 (COVID19). https://www.who.int/docs/default-source/coronaviruse/who-china-joint-mission-on-covid-19-fi nal-report.pdf. (Date accessed: May 14, 2020).

22. McNeil Jr, DG. Inside China's all-out war on the coronavirus. https://www.nytimes.com/2020/03/04/health/coronavirus-china-aylward.html. (Date accesse d: May 14, 2020).

23. Maddow R. How a country serious about coronavirus does testing and quarantine. https://www.msnbc.com/rachel-maddow/watch/how-a-country-serious-about-coronavirus-doestesting-and-quarantine-80595013902. (Date accessed: May 14, 2020). 
Figure Legends

Figure 1: Descriptions of the first and second stages by SEIAR and SEIAR-CQFH models

Panel A: Epidemiological descriptions of exposed (E), symptomatic infectious (I) and asymptomatic infectious (A) individuals

Panel B: Descriptions of the first stage (Dec 7 to Jan 22) by SEIAR model

Panel C: Descriptions of the second stage (Jan 23 to March 18) by SEIAR-CQFH model

Figure 2: The number of COVID-19 cases and the basic reproductive number (BRN) simulated by SEIAR and SEIAR-CQFH models

Panel A: Comparison of the number of new cases simulated by model and real data

Panel B: Comparison of the cumulative number of cases simulated by model and real data

Panel C: Comparison of the number of deaths simulated by model and real data

Panel D: The BRN of different types of cases

Figure 3: The cumulative number of COVID-19 cases and deaths varies with the number of beds in Fangcang shelter hospitals

Panel A: The cumulative number of COVID-19 cases

Panel B: The cumulative number of COVID-19 deaths

Figure 4: The cumulative number of COVID-19 cases varies with the number of beds in designated hospitals

Figure 5: The cumulative number of COVID-19 cases and deaths varies with the time intervals

Panel A: The cumulative number of COVID-19 cases varies with the time intervals from illness onset to hospital visit

Panel B: The cumulative number of COVID-19 deaths varies with the time intervals from 
illness onset to hospital visit

Panel C: The cumulative number of COVID-19 cases varies with the time intervals from hospital visit to diagnosis

Panel D: The cumulative number of COVID-19 deaths varies with the time intervals from hospital visit to diagnosis

Figure 6: The cumulative number of COVID-19 cases varies with the joint prevention and control measures 


\section{Figures}

A

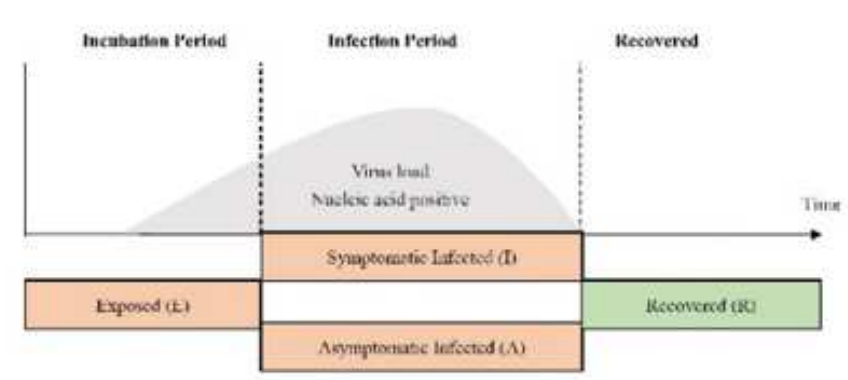

B

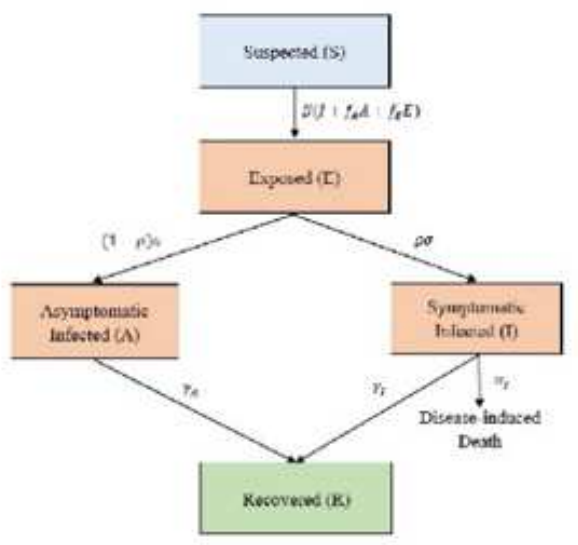

C

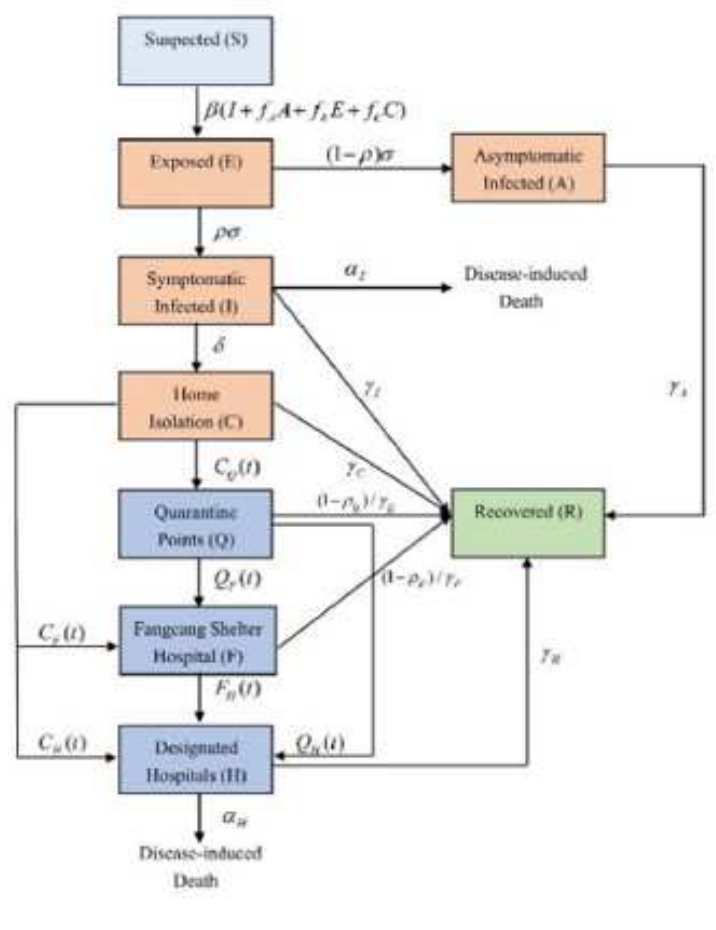

\section{Figure 1}

Descriptions of the first and second stages by SEIAR and SEIAR-CQFH models Panel A: Epidemiological descriptions of exposed (E), symptomatic infectious (I) and asymptomatic infectious (A) individuals Panel B: Descriptions of the first stage (Dec 7 to Jan 22) by SEIAR model Panel C: Descriptions of the second stage (Jan 23 to March 18) by SEIAR-CQFH model 
A

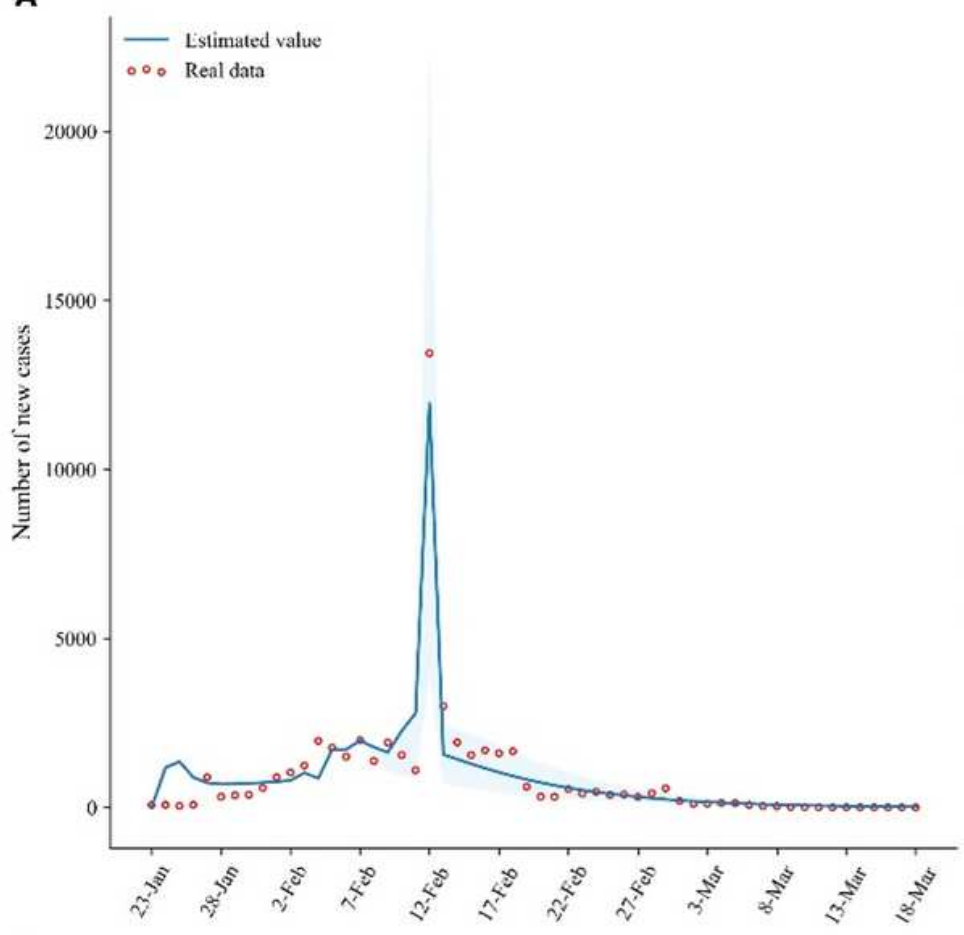

C

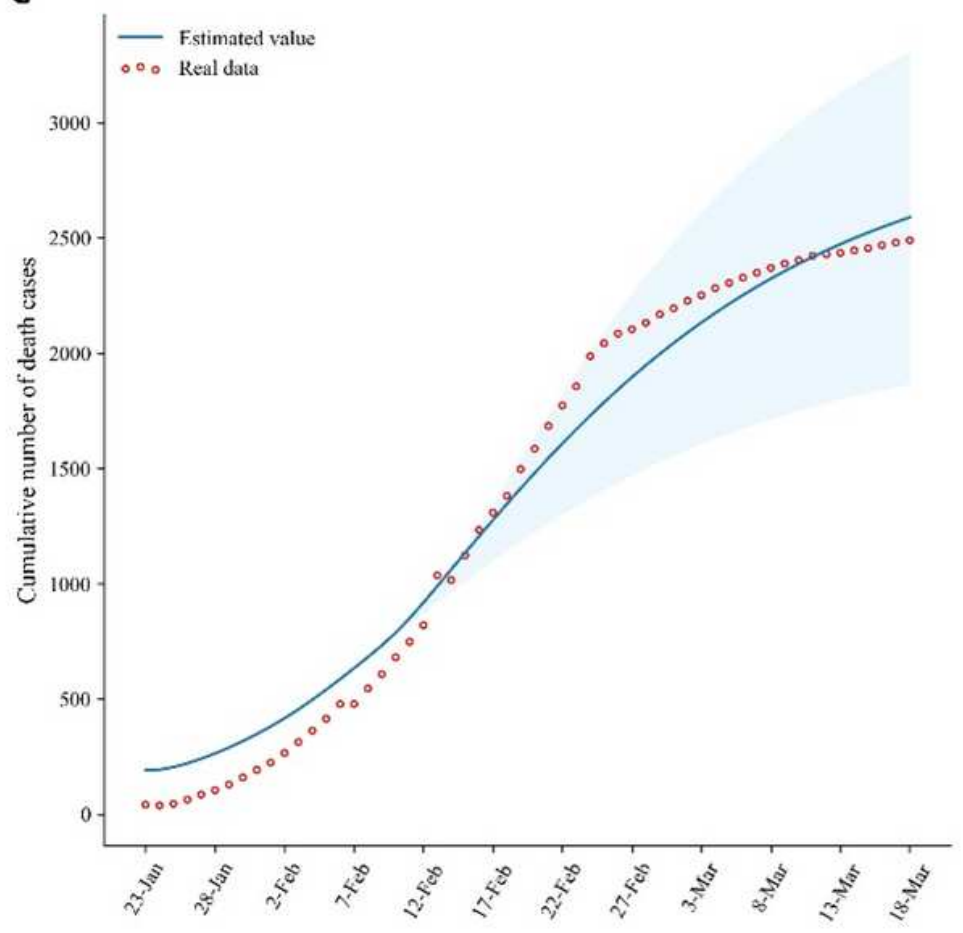

B

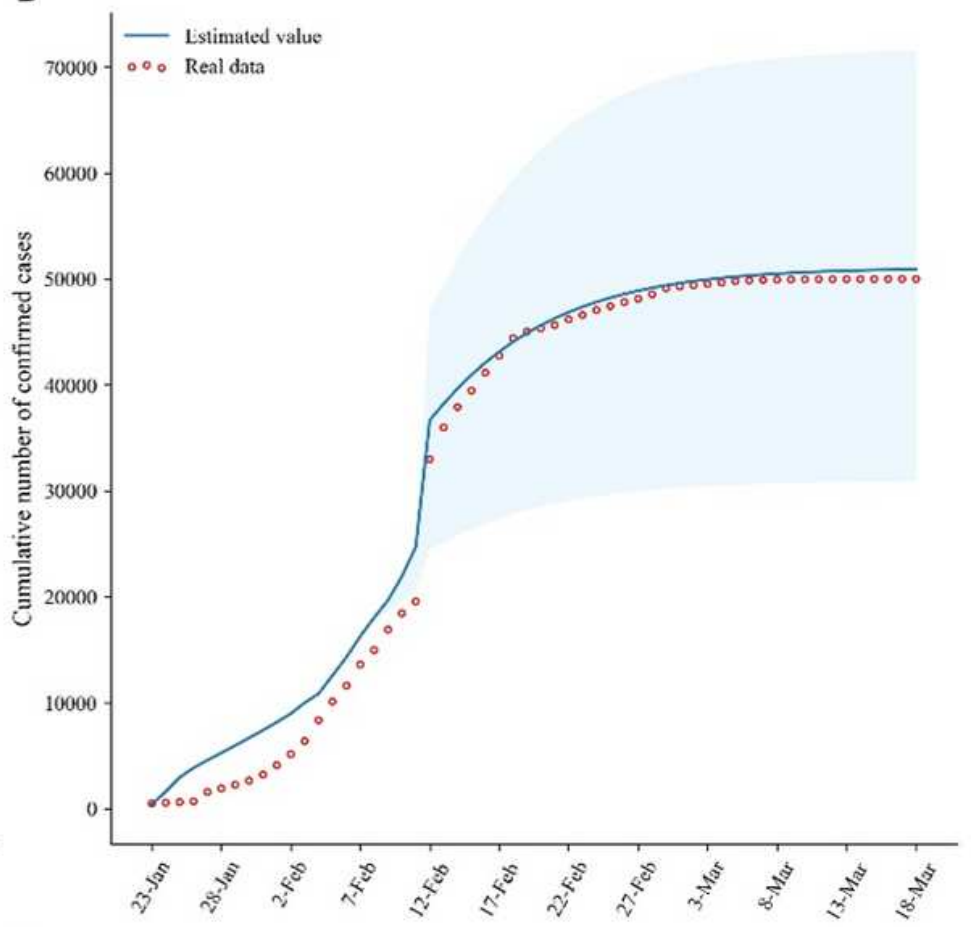

D

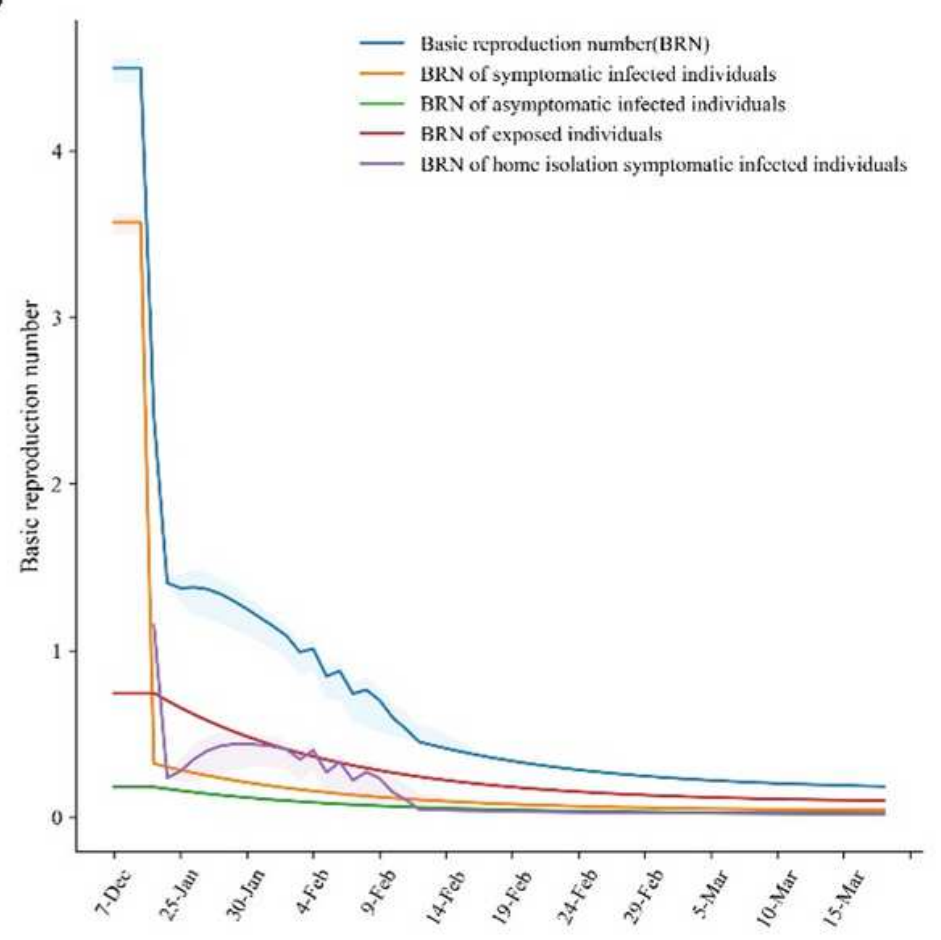

\section{Figure 2}

The number of COVID-19 cases and the basic reproductive number (BRN) simulated by SEIAR and SEIARCQFH models Panel A: Comparison of the number of new cases simulated by model and real data Panel B: Comparison of the cumulative number of cases simulated by model and real data Panel C: Comparison of the number of deaths simulated by model and real data Panel D: The BRN of different types of cases 
A

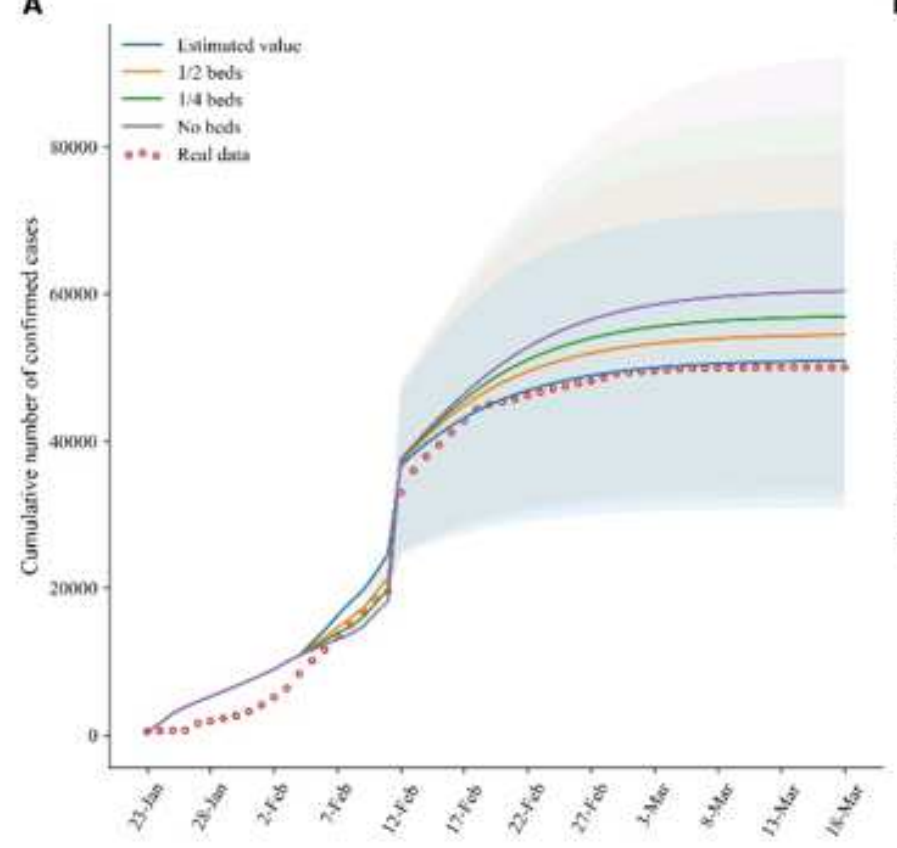

B

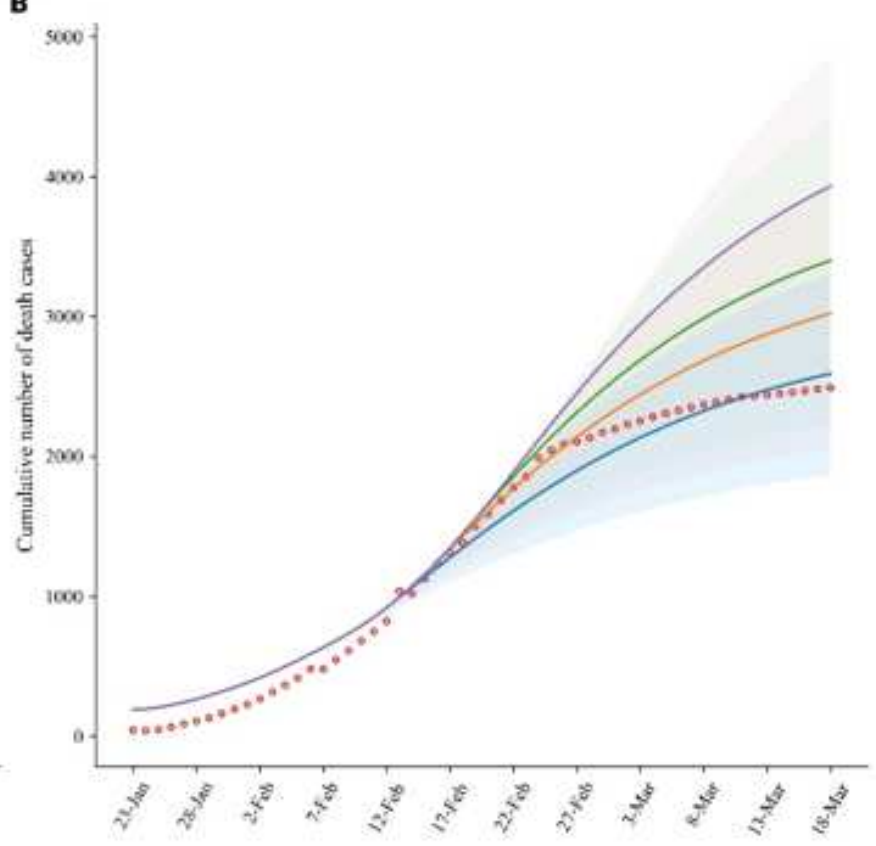

Figure 3

The cumulative number of COVID-19 cases and deaths varies with the number of beds in Fangcang shelter hospitals Panel A: The cumulative number of COVID-19 cases Panel B: The cumulative number of COVID-19 deaths 


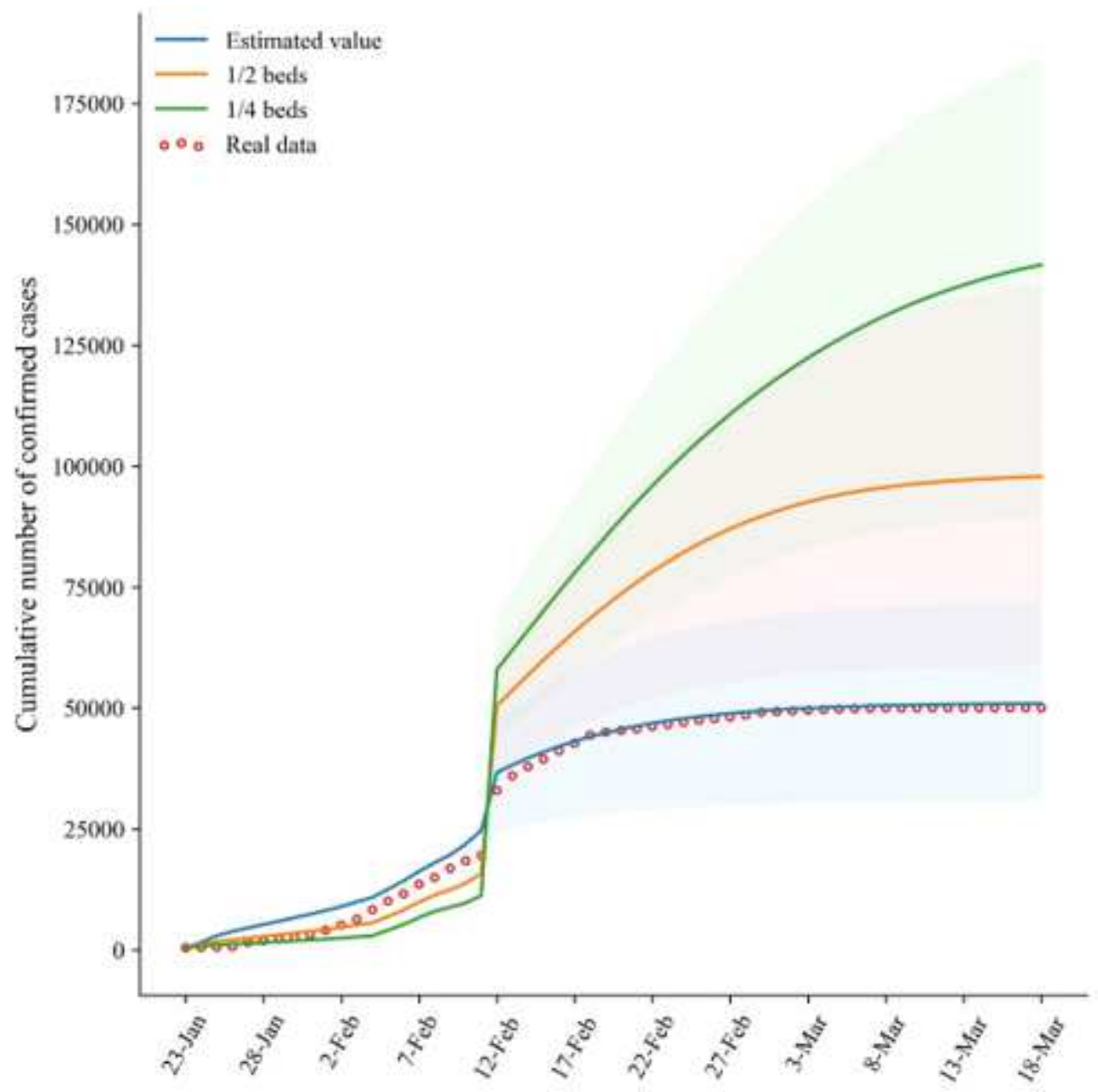

Figure 4

The cumulative number of COVID-19 cases varies with the number of beds in designated hospitals 
A

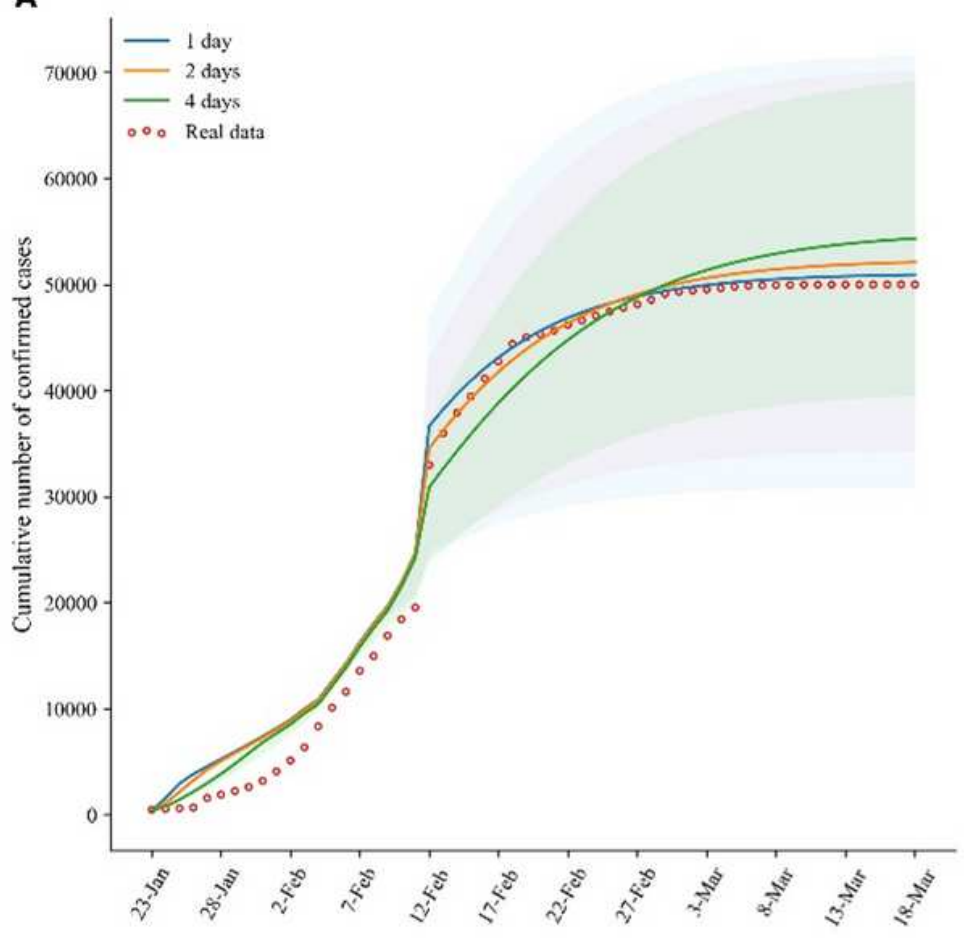

C

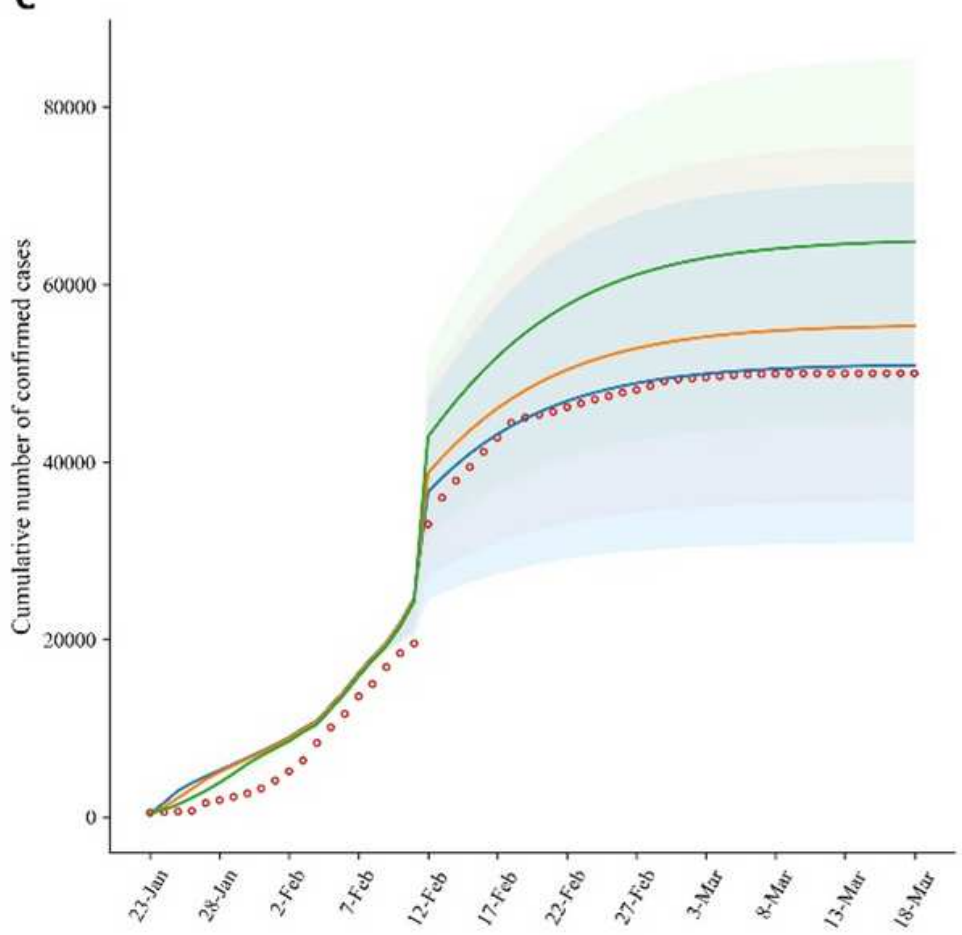

B

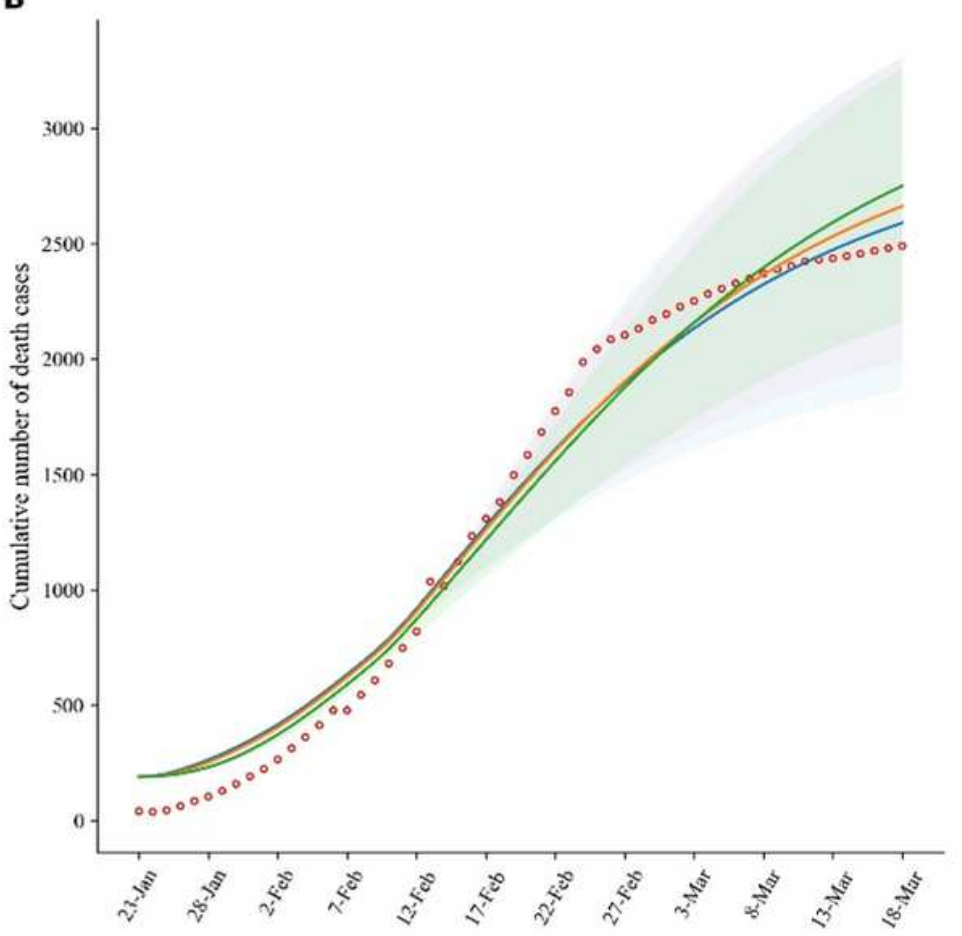

D

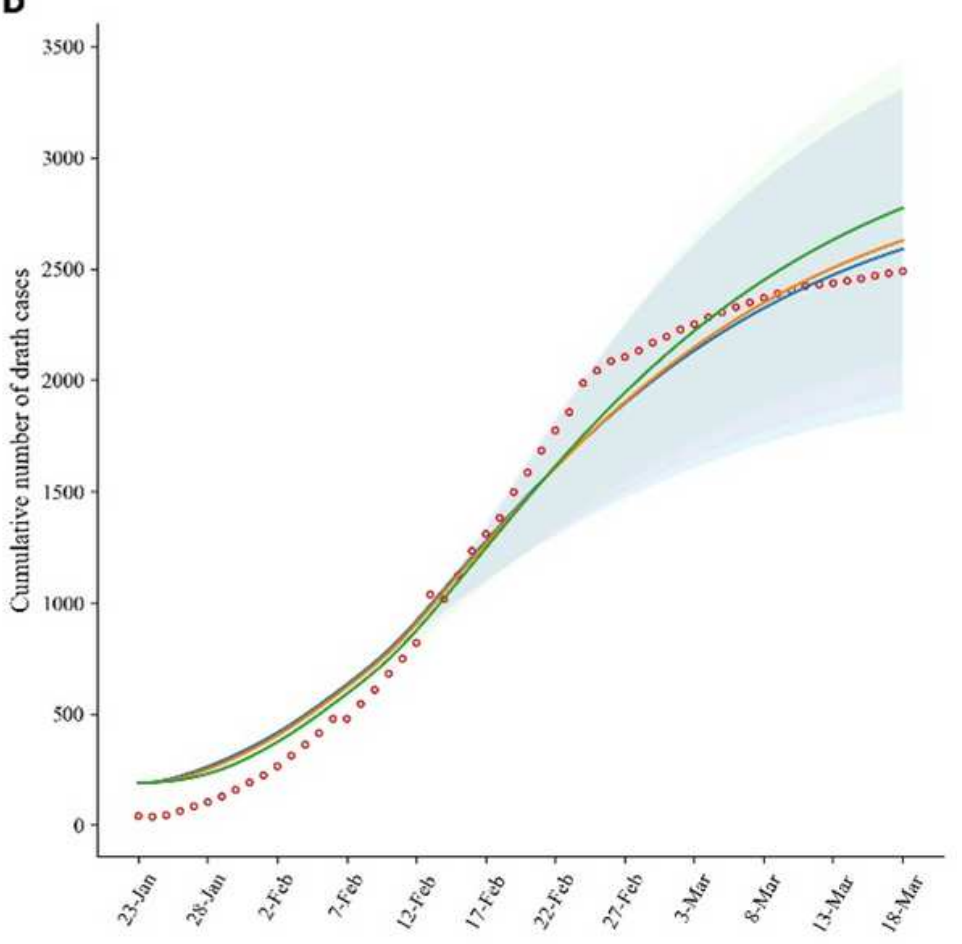

\section{Figure 5}

The cumulative number of COVID-19 cases and deaths varies with the time intervals Panel A: The cumulative number of COVID-19 cases varies with the time intervals from illness onset to hospital visit Panel B: The cumulative number of COVID-19 deaths varies with the time intervals from illness onset to hospital visit Panel C: The cumulative number of COVID-19 cases varies with the time intervals from hospital visit to diagnosis Panel D: The cumulative number of COVID-19 deaths varies with the time intervals from hospital visit to diagnosis 


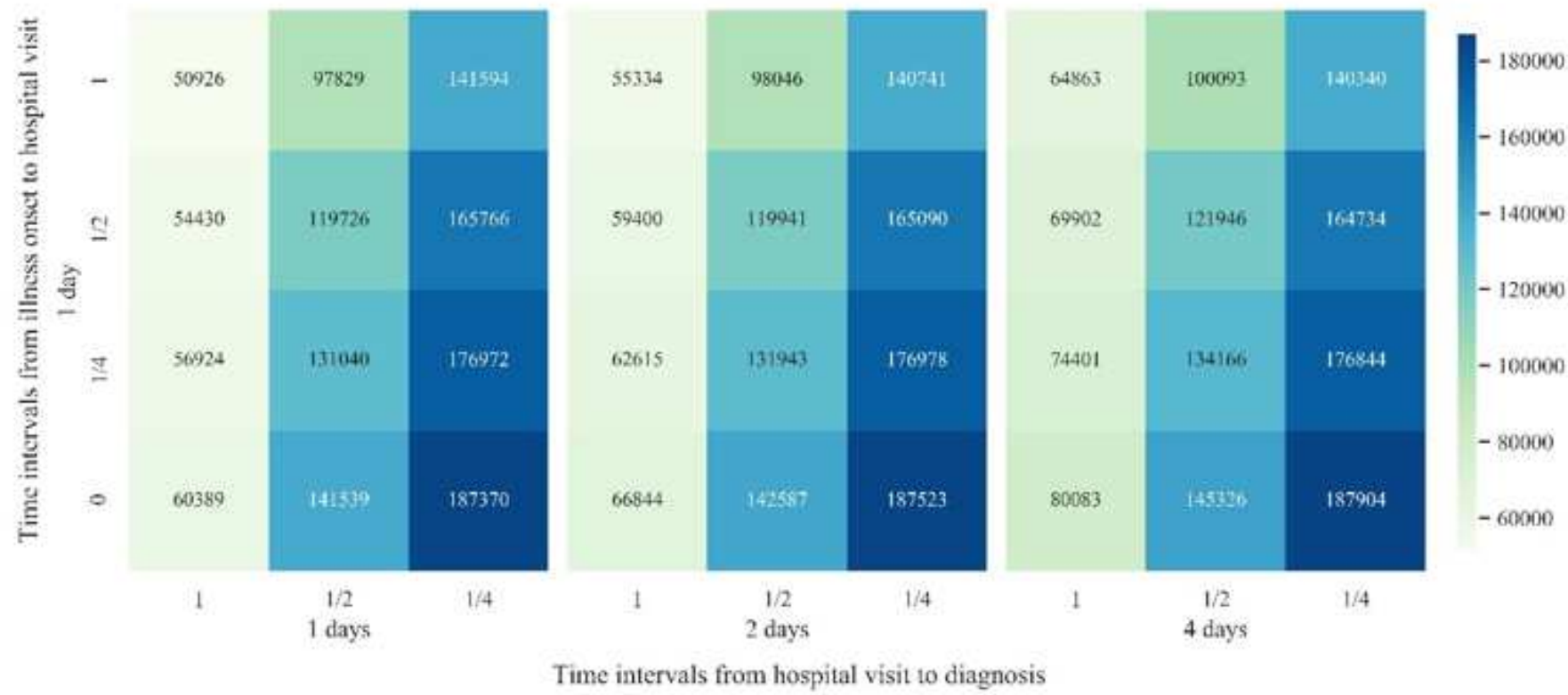

Figure 6

The cumulative number of COVID-19 cases varies with the joint prevention and control measures

\section{Supplementary Files}

This is a list of supplementary files associated with this preprint. Click to download.

- supplementary.docx 